\title{
A Distributionally Robust Joint Chance Constrained Optimization Model for the Dynamic Network Design Problem under Demand \\ Uncertainty
}

\author{
Hua Sun ${ }^{\bullet}$ Ziyou Gao ${ }^{\bullet}$ W.Y. Szeto ${ }^{\bullet}$ Jiancheng Long ${ }^{\bullet}$ Fangxia Zhao ${ }^{\bullet}$
}

\begin{abstract}
This paper develops a distributionally robust joint chance constrained optimization model for a dynamic network design problem (NDP) under demand uncertainty. The major contribution of this paper is to propose an approach to approximate a joint chance-constrained Cell Transmission Model (CTM) based System Optimal Dynamic Network Design Problem with only partial distributional information of uncertain demand. The proposed approximation is tighter than two popular benchmark approximations, namely the Bonferroni's inequality and second-order cone programming (SOCP) approximations. The resultant formulation is a semidefinite program which is computationally efficient. A numerical experiment is conducted to demonstrate that the proposed approximation approach is superior to the other two approximation approaches in terms of solution quality. The proposed approximation approach may provide useful insights and have broader applicability in traffic management and traffic planning problems under uncertainty.
\end{abstract}

Keywords Dynamic network design problem · Distributionally robust joint chance constraints $\cdot$ Worst-case conditional value-at-risk $\cdot$ Semidefinite programming $\cdot$ Demand uncertainty

\section{Introduction}

Traditionally, dynamic transportation network design problems assume that the input data demand and parameters are deterministic. However, in reality, the input data and parameters are usually uncertain. The evaluation of network performance without accounting for the uncertainty can potentially lead to suboptimal network design

Hua Sun

MOE Key Laboratory for Urban Transportation Complex Systems Theory and Technology, Beijing Jiaotong University, Beijing 100044, China

Email: sunhua19800601@163.com

Ziyou Gao

Institute of System Science, School of Traffic and Transportation, Beijing Jiaotong University, Beijing 100044, China

Email: zygao@bjtu.edu.cn

W.Y. Szeto

Department of Civil Engineering, The University of Hong Kong, Pokfulam Road, Hong Kong, China

Email: ceszeto@hku.hk

Jiancheng Long

School of Transportation Engineering, Hefei University of Technology, Hefei 230009, China

Email: jianchenglong@hfut.edu.cn

Fangxia Zhao

MOE Key Laboratory for Urban Transportation Complex Systems Theory and Technology, Beijing Jiaotong University , Beijing 100044, China

Email: 11114204@bjtu.edu.cn 
decisions (Waller et al. 2001). Thus, it is of paramount importance to study the dynamic network design problem under uncertainty from a pragmatic perspective. Recently, chance-constrained programming (CCP) (Charnes et al. 1958) has been employed to formulate and analyze the dynamic NDP under uncertainty (Waller and Ziliaskopoulos 2001; Ukkusuri and Waller 2008) or the core problem of dynamic NDPs, i.e., dynamic traffic assignment (DTA), (Waller and Ziliaskopoulos 2006; Yazici and Ozbay 2010; Chung et al. 2012). In these studies, except Chung et al. (2012), the exact probability distributions of uncertainty are assumed to be known perfectly. In fact, these distributions may be unavailable (inaccurate) as we may have no (insufficient) data to calibrate the distributions, but only the partial information on the distribution, such as its first and second moments and its support, may be available. Against this background, a distributionally robust chance-constrained approach has been introduced recently to formulate and approximate DTA (Chung et al. 2012). However, the approximation for distributionally chance constraints in Chung et al. (2012) is overly conservative.

In this paper, we propose a computationally tractable and less conservative approximation method for formulating the chance-constrained system optimal dynamic network design problem based on known mean and variance of the uncertain time-dependent traffic demand, aiming to provide a robust and tractable but less conservative framework for the dynamic traffic planning and control. A numerical example is presented in this paper to illustrate the value of our less conservative approximation scheme in the context of stochastic dynamic NDPs. Moreover, computational validity is also demonstrated in the proposed framework. This paper further enriches the body of knowledge in stochastic dynamic NDPs by considering the issues in relation to the robustness, conservatism and partial distributional information.

\section{Literature Review}

The transportation network design problem is at the core of many transportation applications. The models and algorithms have been extensively studied in the past three decades (Boyce 1984; Magnanti and Wong 1984; Minoux 1989; Yang and Bell 1998; Chen et al. 2011). However, the vast body of previous literature has focused only on the static NDPs. Lin et al. (2011) pointed out that static NDP models cannot capture realistic, time-varying demand whereas the dynamic NDP models can. Janson (1995) and Waller (2000) revealed that the DTA-based NDP models are more desirable than the static models. In order to overcome these deficiencies, a variety of recent papers focused more on the DTA-based NDPs (Waller et al. 2006; Ukkusuri and Waller 2008; Karoonsoontawong and Waller 2006). According to Lin et al. (2011), these DTA-based NDP models can be divided into two categories: 1) single-level models and 2) bi-level models.

The single-level DTA-based NDP models are those based on the single-destination system-optimal (SO) (Ziliaskopoulos 2000) or user-optimal (UO) (Ukkusuri and Waller 2008) dynamic traffic assignment models, including the single-level SO DTA-based NDP model formulated by Waller et al. (2006) and the single-level SO DTA-based NDP model formulated by Ukkusuri and Waller (2008). The common feature of the above-mentioned single-level models is that the cell transmission model (CTM) (Daganzo 1994, 1995) is 
used to model the dynamic traffic flow propagation, and the demand is assigned to the network by either the dynamic SO or UO principle. Moreover, these models are often formulated as a linear program, and therefore they are computationally tractable.

Similar to the static NDPs, the bi-level DTA-based NDPs can usually be modeled as leader-follower games, in which the transportation manager is the leader of each game who makes network design decisions and the users are the followers of the game who can freely choose their route (Boyce 1984; Yang and Bell 1998). Therefore, each of the bi-level DTA-based NDP models can be formulated as a bi-level linear program, in which the objective of the upper-level model is to minimize the total system travel time, whereas the lower-level characterizes the dynamic UO flow pattern. An example of the bi-level DTA-based NDP model is the model developed by Karoonsoontawong and Waller (2006). It is noted that although a bi-level DTA-based NDP model can be reformulated into a single-level model, the resultant formulation is a mathematical program with equilibrium constraints (MPEC) (or equivalently a mathematical program with complementarity constraints (MPCC)) rather than a linear program. Therefore, the bi-level models are more difficult to solve than the single-level models. Hence, heuristics are often developed to solve the bilevel models for general network settings. For example, Karoonsoontawong and Waller (2006) developed three meta-heuristics, namely simulation annealing, genetic algorithm, and random search, for solving the DTA-based NDPs over multi-destination large-size networks. Lin et al. (2011) and Lin (2011) further proposed two heuristic algorithms based on the Dantzig-Wolfe decomposition principle and the dual variable approximation for solving the bi-level DTA-based NDPs.

On the other hand, uncertainty is inseparable from transportation problems. For example, travel demand is uncertain. Without an explicit and rigorous recognition of the uncertainty, any transportation network development plans and policies may take on unnecessary risk and even result in misleading outcomes (Zhao and Kockelman 2002). It is therefore important to capture uncertainty in NDPs. In this regard, a variety of recent papers introduced the uncertainty in the CTM-based NDP or DTA models.

The general approaches of addressing uncertainty in CTM-based NDP or DTA studies include chance-constrained programming (CCP) (Waller and Ziliaskopoulos 2001; Waller and Ziliaskopoulos 2006; Ukkusuri and Waller 2008), two-stage stochastic linear programming with recourse (SLP2) (Waller and Ziliaskopoulos 2001; Karoonsoontawong and Waller 2007; Patil and Ukkusuri 2007; Ukkusuri and Waller 2008) and robust optimization (RO) (Karoonsoontawong and Waller 2006; Karoonsoontawong and Waller 2007; Karoonsoontawong and Waller 2010; Chung et al. 2011; Ben-Tal et al. 2011). The first two approaches have been adopted since early 20s. For example, Waller and Ziliaskopoulos (2001) applied CCP and SLP2 to formulate the single-level CTM-based SO NDP with stochastic demands. Moreover, Ukkusuri and Waller (2008) introduced the single-level UO versions of CCP and SLP2 models, and compared them with the corresponding SO versions. Yazici and Ozbay (2010) further proposed a CTM-based DTA model with probabilistic demand and road capacity constraints. Karoonsoontawong and Waller (2007) applied SLP2 to formulate the bi-level CTM-based NDPs. Karoonsoontawong and Waller (2010) further extended their previous model to incorporate the signal setting design decision. The above-mentioned CTM-based NDP 
models were, however, developed by the CCP or SLP2 approach and it is necessary for the model users to know the probability distributions of the uncertain input data and parameters in order to use these models. In fact, the distributions may be unavailable (inaccurate) as we may have no (insufficient) data to calibrate the distributions. Therefore, robust optimization (Mulvey et al. 1995; Ben-Tal et al. 2009; Bertsimas et al. 2011) have been introduced recently to address the limitations of CTM-based NDPs or DTA.

According to Chung et al. (2012), robust optimization can be roughly classified into two groups: 1) scenario-based robust optimization, and 2) set-based robust optimization. The scenario-based robust optimization approach represents uncertainty via a limited number of discrete scenarios associated with strictly positive probabilities of occurrence, and attempts to solve the optimization problem across these scenarios for solutions that are near-optimal with respect to the population of all possible realizations of uncertainty (Yin et al. 2009). Mulvey et al. (1995) developed a scenario-based RO approach for general linear programming (LP) problems. Karoonsoontawong and Waller (2006) adopted this approach to propose the CTM-based NDP bi-level linear programming formulation. Karoonsoontawong and Waller (2007) adopted the same approach to formulate the CTM-based single-level SO and UO NDP models and bi-level NDP model, and made comparison with SPL2 and deterministic approaches. Ukkusuri et al. (2007) adopted the bi-level programming approach to develop a scenario-based robust discrete network design model, in which the lower-level problem is the dynamic user equilibrium problem. Karoonsoontawong and Waller (2010) later presented a scenario-based robust bi-level model for the combined network capacity expansion and signal setting design problem. Meanwhile, Mudchanatongsuk et al. (2008) pointed out that the scenario-based robust optimization approach has the following three difficulties: 1) Similar to CCP and SLP2, the scenario-based robust optimization approach also requires that the probability distribution of each scenario is known in advance; 2) the numerous scenarios used in accurately representing the uncertainty can lead to large, computationally challenging problems, and; 3) the solution obtained may be sensitive to possible uncertainty outcomes. Therefore, more attention has been paid to the set-based robust optimization approach recently.

Unlike CCP, SLP2 and the scenario-based robust optimization approach, the set-based robust optimization approach (Kouvelis and Yu 1997; Ben-Tal and Nemirovski 1998, 1999, 2000, 2002; Bertsimas and Sim 2004) does not require the assumption that the probability distributions of the uncertain input data and parameters are known. Therefore, the set-based robust optimization approach recently has not only been applied to the static NDPs (Yin and Lawpongpanich 2007, 2008; Yin et al. 2009; Lou et al. 2009; Lou et al. 2010) but also the CTM-based dynamic NDPs (Chung et al. 2011) or DTA (Yao et al. 2009). In these studies, the uncertain input data and parameters are assumed to be belonging to a bounded set. For example, Chung et al. (2011) assumed a box uncertain set for demand to formulate a single-level robust NDP model whereas Yao et al. (2009) adopted the polyhedral, box, and ellipsoid uncertain sets for demand to develop the CTM-based system-optimal DTA (SODTA) models.

The robust solutions obtained in the above-mentioned studies are, however, overly conservative. To alleviate the conservatism of the robust solutions, Ben-Tal et al. (2004) 
proposed the adjustable robust optimization approach for general linear programming models. Ben-Tal et al. (2011) used the adjustable robust optimization methodology to solve the CTM-based SODTA under demand uncertainty. The polyhedral set is used as the uncertain demand set and the affinely adjustable robust counterpart (AARC) is reformulated into a linear program by using the affine control rule. Recently, a new robust optimization approach for the chance constraints has been applied to formulate CTM-based SODTA. Chung et al. (2012) developed a CTM-based SODTA model under demand uncertainty with the distributionally robust joint chance constraints. Providing that only the partial distribution information (mean and variance) was available, the distributionally robust joint chance constraints were approximated by the linear constraint based on Bonferroni's inequality. Nevertheless, the Bonferroni's approximation may still be overly conservative.

In this paper, a new approximation approach for the distributionally robust joint chance constraints is proposed to formulate a single-level CTM-based system-optimal NDP (SONDP) under demand uncertainty where only the partial distributional information (i.e., mean and variance) of uncertain demand is available. The single-level structure is adopted because it can provide an easier way to approximate the distributional robust joint chance constraints and makes the resultant NDP model to be computationally tractable. We develop a less conservative approximation for the distributionally robust joint chance constraints in the context of CTM-based SONDP. The distributionally robust joint chance constraints in the model are firstly approximated by the Worst-Case Conditional Value-at-Risk (WCVaR) constraints, and then the approach proposed by Zymler et al. (2013) is adopted to reformulate the WCVaR constraint into the semidefinite programming (SDP) constraint. The numerical results are provided in the latter section to illustrate the improved solution quality offered by the SDP-based approximation over the two other approximations, i.e., Bonferroni's approximation and the approximation by Chen et al. (2010).

The remainder of this paper is structured as follows. In Section 3, we present a deterministic CTM-based SONDP formulation and reformulate it into a robust joint chance-constrained program after incorporating the uncertain demand. Section 4 presents the Worst-Case CVaR approximation and the other two approximation approaches for the robust joint chance constraints. The solution algorithm for solving the resultant program derived from the SDP and SOCP approximations is presented in Section 5. In Section 6, the numerical experimental results are presented to demonstrate the effectiveness of the proposed approach. Finally, Section 7 concludes the paper and proposes the direction for future research.

\section{Deterministic and distributionally robust joint chance constraint model}

In this section, we firstly describe a deterministic CTM-based system-optimal NDP (SONDP) and then reformulate it into a robust joint chance-constrained program by introducing the uncertain demand. For ease of discussion, the notation used in these models is presented in Table 1.

Table 1 Notations 


\begin{tabular}{|c|c|}
\hline Sets & Description \\
\hline $\mathfrak{J}$ & Set of time intervals $\{1,2, \ldots, T\}$ \\
\hline C & Set of cells \\
\hline$C_{R}$ & Set of source cells \\
\hline$C_{S}$ & Set of sink cells \\
\hline \multirow{2}{*}{$A$} & Adjacent matrix, $A=\left\{a_{i j}\right\}$; if cell $i$ is connected to cell $j$, then $a_{i j}=1$, \\
\hline & otherwise $a_{i j}=0$ \\
\hline Parameters & Description \\
\hline$d_{i}^{t}$ & Demand generated at cell $i$ during time interval $t, i \in C_{R}$ \\
\hline$c_{i}^{t}$ & Travel cost associated with a vehicle in cell $i$ during time interval $t$ \\
\hline$N_{i}^{t}$ & Capacity of cell $i$ during time interval $t$ \\
\hline$\delta_{i}^{t}$ & $\begin{array}{l}\text { Ratio of the free-flow speed to the backward wave speed associated with } \\
\text { cell } i \text { and time interval } t\end{array}$ \\
\hline$Q_{i}^{t}$ & Inflow/Outflow capacity of cell $i$ in time interval $t$ \\
\hline$\hat{x}_{i}$ & Initial number of vehicles in cell $i$ \\
\hline Functions & Description \\
\hline$\chi_{i}$ & Increase in $N_{i}^{t}$ for a unit increase in $b_{i}$ \\
\hline$\phi_{i}$ & Increase in $Q_{i}^{t}$ for a unit increase in $b_{i}$ \\
\hline$B$ & Total budget \\
\hline Variables & Description \\
\hline$b_{i}$ & Budget spent on cell $i$ for improvement \\
\hline$x_{i}^{t}$ & Number of vehicles in cell $i$ in time interval $t$, \\
\hline$y_{i j}^{t}$ & Number of vehicles that move from cell $i$ to cell $j$ during time interval \\
\hline & 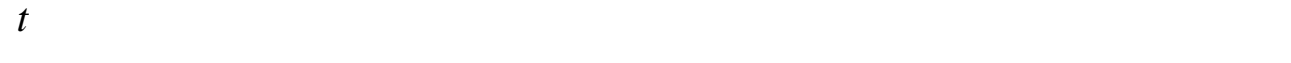 \\
\hline$b$ & Vector of budgets allocated to cells, $b=\left(\ldots ., b_{i}, \ldots\right)$ \\
\hline$x$ & Vector of the numbers of vehicles in cells, $x=\left(\ldots, x_{i}^{t}, \ldots\right)$ \\
\hline$y$ & Vector of the numbers of vehicles moving between two adjacent cells, \\
\hline
\end{tabular}




$$
y=\left(\ldots, y_{i j}^{t}, \ldots\right)
$$

The CTM-based SONDP formulation aims to minimize the total cost, which is the sum of the product of the number of vehicles in each cell in each time interval and the corresponding travel cost. The travel cost of a vehicle in cell $i$ during time interval $t$, $c_{i}^{t}$, is set as follows:

$$
c_{i}^{t}= \begin{cases}1 & i \in C \backslash C_{s}, t \neq T, \\ M & i \in C \backslash C_{s}, t=T,\end{cases}
$$

where $M$ is assumed to be a sufficiently large positive number, which can be interpreted as the cost of a vehicle that cannot arrive at the destination by the end of time horizon. Because the penalty cost $M$ is used in the objective function, the objective of the problem can be interpreted as minimizing the number of vehicles staying in the network by the end of the modeling horizon. By assuming a linear relationship between the budget spent on a cell and the additional capacity of that cell, the deterministic CTM-based SONDP can be formulated as the following linear program (Waller et al., 2006):

$$
\text { SONDP: } \min _{x, y, b} \sum_{t \in \Im} \sum_{i \in C \backslash C_{s}} c_{i}^{t} x_{i}^{t} \text {, }
$$

$$
\begin{array}{ll}
x_{i}^{t}-x_{i}^{t-1}-\sum_{k \in C} a_{k i} y_{k i}^{t-1}+\sum_{j \in C} a_{i j} y_{i j}^{t-1}=d_{i}^{t-1} & \forall i \in C_{R}, t \in \mathfrak{I}, \\
x_{i}^{t}-x_{i}^{t-1}-\sum_{k \in C} a_{k i} y_{k i}^{t-1}+\sum_{j \in C} a_{i j} y_{i j}^{t-1}=0 & \forall i \in C \backslash C_{R} \cup C_{S}, t \in \mathfrak{I}, \\
\sum_{j \in C} a_{i j} y_{i j}^{t} \leq x_{i}^{t} & \forall i \in C \backslash C_{S}, t \in \mathfrak{I}, \\
\sum_{k \in C} a_{k i} y_{k i}^{t} \leq \delta_{i}^{t}\left(N_{i}^{t}+\chi_{i} b_{i}-x_{i}^{t}\right) & \forall i \in C \backslash C_{R} \cup C_{S}, t \in \mathfrak{I}, \\
\sum_{k \in C} a_{k i} y_{k i}^{t} \leq Q_{i}^{t}+\phi_{i} b_{i} & \forall i \in C \backslash C_{R} \cup C_{S}, t \in \mathfrak{I}, \\
\sum_{j \in C} a_{i j} y_{i j}^{t} \leq Q_{i}^{t}+\phi_{i} b_{i} & \forall i \in C \backslash C_{S}, t \in \mathfrak{I}, \\
\sum_{i \in C \backslash C_{s}} b_{i} \leq B, & \\
x_{i}^{0}=\hat{x}_{i} & \forall i \in C \backslash C_{S}, \\
y_{i j}^{0}=0, & \forall(i, j) \in C \times C, \\
x_{i}^{t} \geq 0, & \forall i \in C \backslash C_{S}, t \in \mathfrak{I}, \\
y_{i j}^{t} \geq 0, & \forall(i, j) \in C \times C, t \in \mathfrak{I}, \\
b_{i} \geq 0, & \forall i \in C \backslash C_{S} . \\
& \\
& \\
& \\
& \\
& \\
& \\
&
\end{array}
$$


The objective function of SONDP represents the total travel cost, which provides an optimistic estimate or lower bound of total cost as it simplifies the original CTM model by Daganzo (1994, 1995) and allows vehicle holding. Both constraints (1) and (2) are the flow conservation constraints in cell $i$ in time interval $t$. Because only the source cells generate demand, the right-hand-side of constraint (1) is set as $d_{i}^{t-1}$ and the right-hand-side of constraint (2) is equal to zero. Constraint (3) bounds the total outflow rate of a cell by its current occupancy. Constraint (4) ensures that the total inflow rate of a cell is bounded by its remaining capacity. Constraints (5) and (6) state that the total inflow into and outflow rate from a cell are limited by the inflow and outflow capacities respectively. Constraint (7) is a budgetary constraint. The remaining constraints (8) to (12) represent the initial conditions and non-negativity conditions.

As the problem is a minimization problem and constraint (1) is the only set of constraints related to demand generation, constraint (1) can be reformulated into the following inequality constraint (Waller and Ziliaskopoulos 2006, Chung et al. 2012):

$$
x_{i}^{t}-x_{i}^{t-1}-\sum_{k \in C} a_{k i} y_{k i}^{t-1}+\sum_{j \in C} a_{i j} y_{i j}^{t-1} \geq d_{i}^{t-1}, \forall i \in C_{R}, t \in \mathfrak{J} .
$$

This model allows vehicle holding (Doan and Ukkusuri, 2012) because constraint (13) is always binding and equation (1) and constraint (13) are equivalent. When we incorporate the uncertain demand into the deterministic CTM-based SONDP model, we reformulate constraint (13) into the following joint chance constraint (14) with a confidence parameter $\varepsilon \in(0,1)$ :

$$
\mathbf{P}\left(x_{i}^{t}-x_{i}^{t-1}-\sum_{k \in C} a_{k i} y_{k i}^{t-1}+\sum_{j \in C} a_{i j} y_{i j}^{t-1} \geq \tilde{d}_{i}^{t-1} \forall i \in C_{R}, t \in \mathfrak{I}\right) \geq \varepsilon,
$$

where $\tilde{d}_{i}^{t-1}$ denotes the random demand variable. The violation of constraint (14) implies that more demand is realized than is used for prediction. According to the assumption that the only partial distribution information of uncertain demand may be available, the joint chance constraint (14) can be reformulated as follows:

$$
\operatorname{Inf}_{\mathbf{P} \in \mathrm{P}} \mathbf{P}\left(x_{i}^{t}-x_{i}^{t-1}-\sum_{k \in C} a_{k i} y_{k i}^{t-1}+\sum_{j \in C} a_{i j} y_{i j}^{t-1} \geq \tilde{d}_{i}^{t-1} \quad \forall i \in C_{R}, t \in \mathfrak{I}\right) \geq \varepsilon,
$$

where $\mathrm{P}$ denotes the set of all probability distributions that are consistent with the know mean and variance of uncertain demand. Then, the CTM-based SONDP with the distributionally robust joint chance constraints can be rewritten as:

$$
\begin{aligned}
\text { SONDP-RJCCP: } & \min _{x, y, b} \sum_{t \in \mathfrak{I}} \sum_{i \in C \backslash C_{s}} c_{i}^{t} x_{i}^{t}, \\
\text { subject to } & \text { constraints (2)-(12) and (15). }
\end{aligned}
$$

\section{Approximation of distributionally robust joint chance constraints}

In this section, we start with using the approximation approach based on semidefinite programming (SDP) proposed by Zymler et al. (2013) to approximate the distributionally robust joint chance constraint (15), and then present the two benchmark approximations 
of constraint (15). One approximation is based on Bonferroni's inequality and the other is based on second-order conic programming (SOCP) (Chen et al. 2010). Finally, we compare the three approximations for the distributionally robust joint chance constraints.

\subsection{The Worst-Case Conditional Value-at-Risk approximation}

Assume that the uncertain demand depends affinely on a random number $\xi \in \square^{1}$, i.e., $\tilde{d}_{i}^{t}=\mu_{i}^{t}+\sigma_{i}^{t} \xi$, where $\mu_{i}^{t}$ and $\left(\sigma_{i}^{t}\right)^{2}$ are denoted as the mean and variance of the demand, respectively. The mean and variance of the random number $\xi$ are, respectively, assumed to be 0 and 1 , i.e., $E(\xi)=0$, and $\operatorname{Var}(\xi)=1$. For notation purposes, we let the following be the second-order moment matrix of $\xi$ :

$$
\Omega=\left[\begin{array}{cc}
\operatorname{Var}(\xi)+E(\xi) & E(\xi) \\
E(\xi) & 1
\end{array}\right]=\left[\begin{array}{ll}
1 & 0 \\
0 & 1
\end{array}\right]
$$

Based on the above setting, Chen et al. (2010) proved that the joint chance constraint (15) can be reformulated into

$$
\operatorname{Inf}_{\mathbf{P} \in \mathbf{P}} \mathbf{P}\left(\max _{i \in C_{R}, t \in \mathfrak{I}}\left\{\alpha_{i}^{t}\left(x_{i}^{t-1}-x_{i}^{t}+\sum_{k \in C} a_{k i} y_{k i}^{t-1}-\sum_{j \in C} a_{i j} y_{i j}^{t-1}+\mu_{i}^{t-1}+\sigma_{i}^{t-1} \xi\right)\right\} \leq 0\right) \geq \varepsilon,
$$

where $\alpha \in \mathrm{A}=\left\{\alpha_{i}^{t} \mid \alpha \in \square^{\left|C_{R}\right| \times|\Im|}, \alpha=\left(\ldots, \alpha_{i}^{t}, \ldots\right)>0\right\}$ is called the scaling parameter. The choice of $\alpha \in \mathrm{A}$ does not affect the feasible region of the chance constraint (15). Although these scaling parameters are seemingly redundant, it turns out that they can be tuned to improve the quality of approximation. Chen et al. (2010) indicated that constraint (16) represents a distributionally robust individual chance constraint, which can be approximated by a Worst-Case CVaR constraint. Thus, the feasible region of constraint (15) can be approximated by

$$
\mathrm{Z}(\alpha)=\left\{(x, y): \sup _{\mathbf{P} \in \mathrm{P}} \mathrm{CVaR}_{1-\varepsilon}\left(\max _{i \in C_{R}, t \in \mathfrak{I}}\left\{\alpha_{i}^{t}\left(x_{i}^{t-1}-x_{i}^{t}+\sum_{k \in C} a_{k i} y_{k i}^{t-1}-\sum_{j \in C} a_{i j} y_{i j}^{t-1}+\mu_{i}^{t-1}+\sigma_{i}^{t-1} \xi\right)\right\} \leq 0\right\},\right.
$$

Where $\operatorname{CVaR}_{1-\varepsilon}\left(\max _{i \in C_{R}, t \in \mathfrak{I}}\left\{\alpha_{i}^{t}\left(x_{i}^{t-1}-x_{i}^{t}+\sum_{k \in C} a_{k i} y_{k i}^{t-1}-\sum_{j \in C} a_{i j} y_{i j}^{t-1}+\mu_{i}^{t-1}+\sigma_{i}^{t-1} \xi\right)\right\}\right)=$

$$
\inf _{\beta \in \mathbf{R}}\left\{\beta+\frac{1}{1-\varepsilon} \mathbf{E}_{\mathbf{P}}\left(\left[\max _{i \in C_{R}, t \in \mathfrak{I}}\left\{\alpha_{i}^{t}\left(x_{i}^{t-1}-x_{i}^{t}+\sum_{k \in C} a_{k i} y_{k i}^{t-1}-\sum_{j \in C} a_{i j} y_{i j}^{t-1}+\mu_{i}^{t-1}+\sigma_{i}^{t-1} \xi\right)\right\}-\beta\right]^{+}\right)\right\} .
$$

$\beta$ is a decision variable in the chance constraint; $\mathbf{E}_{\mathbf{p}}(\square)$ denotes the expectation with 
respect to $\mathbf{P}$, and $(\bullet)^{+}=\max \{\bullet, 0\}$ (Rockafellar and Uryasev 2000, 2002). In contrast to the chance constraint (16), $(x, y) \in \mathrm{Z}(\alpha)$ depends on the choice of $\alpha \in \mathrm{A}$. Because the max function in constraint (16) is not concave, the Worst-Case CVaR constraint (17) is not equivalent to constraint (16) (Chen et al. 2010).

Zymler et al. (2013) developed an approximation approach for distributionally robust chance constraints based on semidefinite programming (SDP). The first- and second-order moments with the supports of uncertain parameters are assumed to be known. Zymler et al. (2013) firstly approximated the distributionally chance constraints by the Worst-case Conditional Value-at-Risk (WCVaR) constraints, and then reformulated the WCVaR constraints into the SDP constraints using the theory of moment problems and conic duality arguments. They argued that this approximation is exact for robust individual chance constraints with concave or quadratic constraint functions and this approximation is tighter than the two other benchmark approximations for robust joint chance constraints. In this study, we adopt their approach to approximate constraint (15) and present the following theorem about the equivalent form of constraint (17).

Theorem 1: If $\tilde{d}_{i}^{t-1}$ follows an unknown probability distribution with the mean $\mu_{i}^{t-1}$ and variance $\left(\sigma_{i}^{t-1}\right)^{2}$, then the distributionally robust joint chance constraint (15) can be approximated by the following semidefinite programming constraint:

$$
\mathrm{Z}(\alpha)=\left\{\begin{array}{c}
\exists(\beta, M M) \in \mathbf{R} \times \mathbf{S}^{2} \\
\beta+\frac{1}{1-\varepsilon}\langle\Omega, M M\rangle \leq 0, M M \pm 0 \\
(x, y): \quad\left[\begin{array}{cc}
0 & \alpha_{i}^{t} \sigma_{i}^{t-1} / 2 \\
\alpha_{i}^{t} \sigma_{i}^{t-1} / 2 & \alpha_{i}^{t}\left(x_{i}^{t-1}-x_{i}^{t}+\sum_{k \in C} a_{k i} y_{k i}^{t-1}-\sum_{j \in C} a_{i j} y_{i j}^{t-1}+\mu_{i}^{t-1}\right)-\beta
\end{array}\right] \pm 0
\end{array}, \forall i \in C_{R}, t \in \mathfrak{I}\right\},
$$

where $\mathbf{S}^{2}$ denotes the space of real symmetric matrices of dimension two, $\langle A, B\rangle=\operatorname{trace}(A B)$ is a trace scalar product of matrices $A$ and $B$, and $A \pm 0$ means that the matrix $A$ is semidefinite.

Proof: We note that constraint (17) is equivalent to $J(x, y, \alpha) \leq 0$, where

$$
\begin{aligned}
J(x, y, \alpha)= & \sup _{\mathbf{P} \in \mathbf{P}} \operatorname{CVaR}_{1-\varepsilon}\left(\max _{i \in C_{R}, t \in \mathfrak{I}}\left\{\alpha_{i}^{t}\left(x_{i}^{t-1}-x_{i}^{t}+\sum_{k \in C} a_{k i} y_{k i}^{t-1}-\sum_{j \in C} a_{i j} y_{i j}^{t-1}+\mu_{i}^{t-1}+\sigma_{i}^{t-1} \xi\right)\right\}\right) \\
= & \sup _{\mathbf{P} \in \mathbf{P}} \inf _{\beta \in \mathbf{R}}\left\{\beta+\frac{1}{1-\varepsilon} \mathbf{E}_{\mathbf{P}}\left(\left[\max _{i \in C_{R}, t \in \mathfrak{I}}\left\{\alpha_{i}^{t}\left(x_{i}^{t-1}-x_{i}^{t}+\sum_{k \in C} a_{k i} y_{k i}^{t-1}-\sum_{j \in C} a_{i j} y_{i j}^{t-1}+\mu_{i}^{t-1}+\sigma_{i}^{t-1} \xi\right)\right\}-\beta\right]^{+}\right)\right\} .
\end{aligned}
$$


According to the stochastic saddle point theorem (Shapiro and Kleywegt, 2002), we can interchange the maximization and minimization operations as below:

$$
J(x, y, \alpha)=\inf _{\beta \in \mathbf{R}}\left\{\beta+\frac{1}{1-\varepsilon} \sup _{\mathrm{P} \in \mathrm{P}} \mathbf{E}_{\mathbf{P}}\left(\left[\max _{i \in C_{R}, t \in \mathfrak{I}}\left\{\alpha_{i}^{t}\left(x_{i}^{t-1}-x_{i}^{t}+\sum_{k \in C} a_{k i} y_{k i}^{t-1}-\sum_{j \in C} a_{i j} y_{i j}^{t-1}+\mu_{i}^{t-1}+\sigma_{i}^{t-1} \xi\right)\right\}-\beta\right]^{+}\right)\right\} .
$$

Next, we derive the dual problem of the following Worst-Case expectation problem:

$$
\sup _{\mathbf{P} \in \mathbf{P}} \mathbf{E}_{\mathbf{P}}\left(\left[\max _{i \in C_{R}, t \in \mathfrak{I}}\left\{\alpha_{i}^{t}\left(x_{i}^{t-1}-x_{i}^{t}+\sum_{k \in C} a_{k i} y_{k i}^{t-1}-\sum_{j \in C} a_{i j} y_{i j}^{t-1}+\mu_{i}^{t-1}+\sigma_{i}^{t-1} \xi\right)\right\}-\beta\right]^{+}\right) .
$$

Using Lemma 1 in Zymler et al. (2013), we have:

$$
\inf _{M M \in \mathrm{S}^{2}}\langle\Omega, M M\rangle \text {, }
$$

subject to

$$
\begin{gathered}
{[\xi, 1]^{T} M M[\xi, 1] \geq \max _{i \in C_{R}, t \in \mathfrak{I}}\left\{\alpha_{i}^{t}\left(x_{i}^{t-1}-x_{i}^{t}+\sum_{k \in C} a_{k i} y_{k i}^{t-1}-\sum_{j \in C} a_{i j} y_{i j}^{t-1}+\mu_{i}^{t-1}+\sigma_{i}^{t-1} \xi\right)\right\}-\beta, \quad \forall \xi \in \square,} \\
M M \pm 0 .
\end{gathered}
$$

We note that the above optimization problem represents a lossless reformulation of the worst-case expectation problem (22). The semi-infinite constraint (24) can be expanded into $\left|C_{R}\right| \times|\mathfrak{I}|$ simpler semi-infinite constraints in the form of

$$
[\xi, 1]^{T} M M[\xi, 1] \geq \alpha_{i}^{t}\left(x_{i}^{t-1}-x_{i}^{t}+\sum_{k \in C} a_{k i} y_{k i}^{t-1}-\sum_{j \in C} a_{i j} y_{i j}^{t-1}+\mu_{i}^{t-1}+\sigma_{i}^{t-1} \xi\right)-\beta, \forall i \in C_{R}, t \in \mathfrak{I}, \xi \in \square .
$$

Constraint (26) can be equivalently expressed as

$$
M M-\left[\begin{array}{cc}
0 & \alpha_{i}^{t} \sigma_{i}^{t-1} / 2 \\
\alpha_{i}^{t} \sigma_{i}^{t-1} / 2 & \alpha_{i}^{t}\left(x_{i}^{t-1}-x_{i}^{t}+\sum_{k \in C} a_{k i} y_{k i}^{t-1}-\sum_{j \in C} a_{i j} y_{i j}^{t-1}+\mu_{i}^{t-1}\right)-\beta
\end{array}\right] \pm 0, \forall i \in C_{R}, t \in \mathfrak{I} \text {. }
$$

Therefore, $\quad J(x, y, \alpha)=\inf _{\beta \in \square} \beta+\frac{1}{1-\varepsilon}\langle\Omega, M M\rangle$,

subject to $M M \in \mathbf{S}^{2}, \quad M M \pm 0$,

$$
M M-\left[\begin{array}{cc}
0 & \alpha_{i}^{t} \sigma_{i}^{t-1} / 2 \\
\alpha_{i}^{t} \sigma_{i}^{t-1} / 2 & \alpha_{i}^{t}\left(x_{i}^{t-1}-x_{i}^{t}+\sum_{k \in C} a_{k i} y_{k i}^{t-1}-\sum_{j \in C} a_{i j} y_{i j}^{t-1}+\mu_{i}^{t-1}\right)-\beta
\end{array}\right] \pm 0, \forall i \in C_{R}, t \in \mathfrak{I} .
$$

Thus, the claim follows.

Using Theorem 1, the SONDP-RJCCP can be approximated to an SDP problem, 
formulated as follows.

$$
\begin{aligned}
& \text { SDP model: } \quad \min _{x, y, b, \alpha} \sum_{t \in \mathfrak{I}} \sum_{i \in C \backslash C_{s}} c_{i}^{t} x_{i}^{t}, \\
& \text { subject to } \quad J(x, y, \alpha) \leq 0,
\end{aligned}
$$

$$
\text { constraints (2)-(12). }
$$

\subsection{The Bonferroni Approximation}

A popular approximation for constraint (15) is based on Bonferroni's inequality. We note that constraint (15) is equivalent to the following:

$$
\begin{aligned}
& \operatorname{Inf}_{\mathbf{P} \in \mathbf{P}} \mathbf{P}\left(x_{i}^{t}-x_{i}^{t-1}-\sum_{k \in C} a_{k i} y_{k i}^{t-1}+\sum_{j \in C} a_{i j} y_{i j}^{t-1} \geq \tilde{d}_{i}^{t-1}, \forall i \in C_{R}, t \in \mathfrak{I}\right) \geq \varepsilon \\
& \quad \Leftrightarrow \sup _{\mathbf{P} \in \mathrm{P}} \mathbf{P}\left(\bigcup_{i \in C_{R}, t \in \mathfrak{I}} x_{i}^{t}-x_{i}^{t-1}-\sum_{k \in C} a_{k i} y_{k i}^{t-1}+\sum_{j \in C} a_{i j} y_{i j}^{t-1}<\tilde{d}_{i}^{t-1}\right) \leq 1-\varepsilon .
\end{aligned}
$$

Moreover, according to Chung et al. (2012), Bonferroni’s inequality implies that

$$
\begin{aligned}
& \mathbf{P}\left(\bigcup_{i \in C_{R}, t \in \mathfrak{I}} x_{i}^{t}-x_{i}^{t-1}-\sum_{k \in C} a_{k i} y_{k i}^{t-1}+\sum_{j \in C} a_{i j} y_{i j}^{t-1}<\tilde{d}_{i}^{t-1}\right) \leq \\
& \sum_{i \in C_{R}, t \in \mathfrak{I}} \mathbf{P}\left(x_{i}^{t}-x_{i}^{t-1}-\sum_{k \in C} a_{k i} y_{k i}^{t-1}+\sum_{j \in C} a_{i j} y_{i j}^{t-1}<\tilde{d}_{i}^{t-1}\right), \forall \mathbf{P} \in \mathrm{P} .
\end{aligned}
$$

Thus, we have

$$
\operatorname{Inf}_{\mathbf{P} \in \mathrm{P}} \mathbf{P}\left(x_{i}^{t-1}-x_{i}^{t}+\sum_{k \in C} a_{k i} y_{k i}^{t-1}-\sum_{j \in C} a_{i j} y_{i j}^{t-1}+\tilde{d}_{i}^{t-1} \leq 0\right) \geq 1-\varepsilon_{i, t}, \forall i \in C_{R}, t \in \mathfrak{I},
$$

where the confidence level $\varepsilon_{i, t}$ is required to satisfy the constraint $\sum_{i \in C_{R}, t \in \mathfrak{I}} \varepsilon_{i, t} \leq 1-\varepsilon$. Therefore, constraint (32) represents the conservative approximation for constraint (15). A major limitation of the Bonferroni approximation is that the approximation quality critically depends on the choice of confidence level $\varepsilon_{i, t}$. Unfortunately, the problem of finding the best $\varepsilon_{i, t}$ for constraint (15) is nonconvex and it is believed to be intractable (Nemirovski and Shapiro 2006). According to Nemirovski and Shapiro (2006), we set $\varepsilon_{i, t}=\frac{1-\varepsilon}{\left|C_{R}\right| \times|\mathfrak{I}|}$, where $\left|C_{R}\right|$ is the number of source cells and $|\mathfrak{I}|$ denotes the number of discrete time intervals. Thus, constraint (32) can be reformulated into the linear constraint as follows (Calafiore and Ghaoui 2006; Chung et al. 2012):

$$
x_{i}^{t-1}-x_{i}^{t}+\sum_{k \in C} a_{k i} y_{k i}^{t-1}-\sum_{j \in C} a_{i j} y_{i j}^{t-1}+\mu_{i}^{t-1}+\sigma_{i}^{t-1} \sqrt{\frac{\left|C_{R}\right| \times|\mathfrak{I}|}{1-\varepsilon}-1} \leq 0, \forall i \in C_{R}, t \in \mathfrak{I} .
$$


Thus, the approximated SONDP-RJCCP can be formulated as following LP model:

$$
\text { LP model: } \quad \min _{x, y, b} \sum_{t \in \mathfrak{I}} \sum_{i \in C \backslash C_{s}} c_{i}^{t} x_{i}^{t} \text {, }
$$

subject to constraints (2)-(12) and (33).

As shown in the above formulation, the model is still an LP model and can be computed efficiently. However, the Bonferroni approximation is overly conservative. Zymler et al. (2013) proved that the accuracy of the Bonferroni approximation diminishes with an increasing number of joint constraints if the inequalities in the joint chance constraints are positively correlated.

\subsection{The approximation by Chen et al. (2010)}

To minimize the over-conservatism of the Bonferroni approximation, Chen et al. (2010) proposed an approximation approach for the robust joint chance constraints based on second-order cone programming (SOCP) by using inequalities from the probability theory. Moreover, they also proved that their approximation is tighter than the Bofferroni approximation. In this subsection, we adopt their results to approximate constraint (15). Similar to the Worst-Case CVaR approximation, according to the above discussion, the robust joint constraint (15) can be approximated by

$$
\hat{Z}(\alpha)=\left\{(x, y): \sup _{\mathbf{P} \in \mathrm{P}} \operatorname{CVaR}_{1-\varepsilon}\left(\max _{i \in C_{R}, t \in \mathfrak{I}}\left\{\alpha_{i}^{t}\left(x_{i}^{t-1}-x_{i}^{t}+\sum_{k \in C} a_{k i} y_{k i}^{t-1}-\sum_{j \in C} a_{i j} y_{i j}^{t-1}+\mu_{i}^{t-1}+\sigma_{i}^{t-1} \xi\right)\right\} \leq 0\right\},\right.
$$

where $\sup _{\mathbf{P} \in \mathrm{P}} \mathrm{CVaR}_{1-\varepsilon}\left(\max _{i \in C_{R}, t \in \mathfrak{I}}\left\{\alpha_{i}^{t}\left(x_{i}^{t-1}-x_{i}^{t}+\sum_{k \in C} a_{k i} y_{k i}^{t-1}-\sum_{j \in C} a_{i j} y_{i j}^{t-1}+\mu_{i}^{t-1}+\sigma_{i}^{t-1} \xi\right)\right\}\right)$

$$
=\inf _{\beta \in \mathbf{R}}\left\{\beta+\frac{1}{1-\varepsilon} \sup _{\mathbf{P} \in \mathrm{P}} \mathbf{E}_{\mathbf{P}}\left(\left[\max _{i \in C_{R}, t \in \mathfrak{I}}\left\{\alpha_{i}^{t}\left(x_{i}^{t-1}-x_{i}^{t}+\sum_{k \in C} a_{k i} y_{k i}^{t-1}-\sum_{j \in C} a_{i j} y_{i j}^{t-1}+\mu_{i}^{t-1}+\sigma_{i}^{t-1} \xi\right)\right\}-\beta\right]^{+}\right)\right\} .
$$

Chen et al. (2010) employed the results of Chen and Sim (2009) to provide an upper bound of $\mathbf{E}_{\mathbf{P}}\left([\bullet]^{+}\right)$. Thus, constraint (34) can be approximated by the following SOCP (Chen et al. 2010):

$$
\hat{J}(x, y, \alpha) \leq 0,
$$

where $\quad \hat{J}(x, y, \alpha)=\min _{w, w^{0} \in \mathbf{R}}\left\{\min _{\beta \in \mathbf{R}}\left[\beta+\frac{1}{1-\varepsilon} \pi\left(w^{0}-\beta, w\right)\right]\right.$

$$
\begin{aligned}
& \left.+\frac{1}{1-\varepsilon}\left[\sum_{i \in C_{R}, t \in \mathfrak{I}} \pi\left(\alpha_{i}^{t}\left(x_{i}^{t-1}-x_{i}^{t}+\sum_{k \in C} a_{k i} y_{k i}^{t-1}-\sum_{j \in C} a_{i j} y_{i j}^{t-1}+\mu_{i}^{t-1}\right)-w^{0}, \alpha_{i}^{t} \sigma_{i}^{t-1}-w\right)\right]\right\} \\
& \text { and } \pi\left(z^{0}, z\right)=\frac{1}{2} z^{0}+\frac{1}{2}\left\|\left(z^{0}, z\right)\right\|_{2} \text {. }
\end{aligned}
$$

Then, the SONDP-RJCCP can be approximated by a second-order cone programming 
problem which can be formulated as follows.

$$
\begin{aligned}
& \text { SOCP model: } \min _{x, y, b, \alpha} \sum_{t \in \mathfrak{J}} \sum_{i \in C \backslash C_{s}} c_{i}^{t} x_{i}^{t}, \\
& \text { subject to } \quad \text { constraints (2)-(12) and (35). }
\end{aligned}
$$

It is noted that similar to the Bonferroni approximation, the approximation proposed by Chen et al. (2010) critically depends on the choice of $\alpha$, and that the problem of finding the best $\alpha$ for the robust joint chance constraint is nonconvex and therefore believed to be intractable.

\section{Solution algorithm}

We adopted the solution algorithm proposed by Chen et al. (2010) to solve the previous SDP and SOCP models. By Theorem 1, the original SONDP-RJCCP can be written as

$$
\begin{aligned}
& \text { SDP: } \quad \min _{x, y, b, \alpha} \sum_{t \in \Im} \sum_{i \in C \backslash C_{s}} c_{i}^{t} x_{i}^{t}, \\
& \text { subject to } \quad J(x, y, \alpha) \leq 0, \\
& \\
& \text { constraints (2)-(12). }
\end{aligned}
$$

Unfortunately, Zymler et al. (2013) proved that the Worst-Case CVaR functional $J(x, y, \alpha)$ in constraint (29) is merely biconvex, but not jointly convex in $(x, y)$ and $\alpha$, and hence the SDP model is nonconvex. However, when the scaling parameters $\alpha$ are fixed, the problem becomes convex and tractable.

To explain the preceding point, we define the set $\overline{\mathrm{A}} \equiv\left\{\alpha: \alpha_{i}^{t} \geq 1, \forall i \in C_{R}, t \in \mathfrak{I}, \sum_{i \in C_{R}, t \in \mathfrak{I}} \alpha_{i}^{t}=M\right\}$, where $M$ is a large number. Obviously, unlike $\mathrm{A}$, the set $\overline{\mathrm{A}}=$ is closed. When $\alpha \in \overline{\mathrm{A}}=$ is fixed, the SDP problem is reduced to:

$$
\begin{array}{ll}
\text { SDP1: } & \min _{x, y, b} \sum_{t \in \mathfrak{I}} \sum_{i \in C \backslash C_{s}} c_{i}^{t} x_{i}^{t}, \\
\text { subject to } & J(x, y, \alpha) \leq 0, \\
& \text { constraints (2)-(12). }
\end{array}
$$

SDP1 is equivalent to a tractable SDP problem and the feasible solution of SDP1 is also feasible to the SONDP-RJCCP.

Chen et al. (2010) proposed an algorithm to improve the objective value by tuning the scaling parameters $\alpha$. Consequently, they introduce the following tractable SDP problem where $(x, y)$ is fixed:

$$
\begin{gathered}
\text { SDP2: } \min _{\alpha} J(x, y, \alpha) \text {, } \\
\text { subject to } \alpha \in \overline{\mathrm{A}} .
\end{gathered}
$$

Let $\left(x^{*}, y^{*}, b^{*}\right)$ be an optimal solution to SDP1 for a given $\alpha \in \bar{A}$. Then, by the 
feasibility of $\left(x^{*}, y^{*}, b^{*}\right)$ to SDP1, it is found that $J\left(x^{*}, y^{*}, \alpha\right) \leq 0$. If $\left(x^{*}, y^{*}\right)$ is fixed, then the optimal scaling parameters $\alpha^{*}$ corresponding to $\left(x^{*}, y^{*}\right)$ can be obtained by solving SDP2. Then, we have

$$
J\left(x^{*}, y^{*}, \alpha^{*}\right) \leq J\left(x^{*}, y^{*}, \alpha\right) \leq 0 .
$$

This inequality implies that the optimal objective value of SDP1 with the input $\alpha^{*}$ is not larger than $\sum_{t \in \mathfrak{I}} \sum_{i \in C \backslash C_{s}} c_{i}^{t} x_{i}^{t^{*}}$. Thus, a sequence of monotonically decreasing objective values can be obtained by solving SDP1 and SDP2 in alternation. This method depends on the availability of an initial feasible solution $\left(x_{\text {init }}, y_{\text {init }}, b_{\text {init }}\right)$ to SDP1. The following is the procedure of their algorithm, referred to as Algorithm 5.1.

\section{Algorithm 5.1}

Step 1: Let $\left(x_{\text {init }}, y_{\text {init }}, b_{\text {init }}\right)$ be a feasible solution of problem SDP1. Set the iteration number $n \leftarrow 1$, the current solution to $\left(x^{0}, y^{0}, b^{0}\right) \leftarrow\left(x_{\text {init }}, y_{\text {init }}, b_{\text {init }}\right)$ and the current objective function to $f^{0} \leftarrow \sum_{t \in \mathfrak{\Im}} \sum_{i \in C \backslash C_{s}} c_{i}^{t} x_{i}^{t 0}$.

Step 2: Solve problem SDP2 with the input $\left(x^{n-1}, y^{n-1}\right)$ and obtain the optimal scaling parameters $\alpha^{*}$. Set $\alpha^{n} \leftarrow \alpha^{*}$.

Step 3: Solve problem SDP1 with the input $\alpha^{n}$ and obtain the optimal solution $\left(x^{*}, y^{*}, b^{*}\right)$. Set $x^{n} \leftarrow x^{*}, y^{n} \leftarrow y^{*}$ and $f^{n} \leftarrow \sum_{t \in \Im} \sum_{i \in C \backslash C_{s}} c_{i}^{t} x_{i}^{t n}$.

Step 4: If $\left(f^{n}-f^{n-1}\right) /\left|f^{n-1}\right| \leq \gamma \quad$ (where $\gamma$ is a given small tolerance), then stop and output $\left(x^{n}, y^{n}, b^{n}\right)$. Otherwise, set $n \leftarrow n+1$ and return to step 2 .

Zymler et al. (2013) proved that the objective values $\left\{f^{n}\right\}$ generated by Algorithm 5.1 is monotonically decreasing sequence if $\left(x_{\text {init }}, y_{\text {init }}, b_{\text {init }}\right)$ is feasible to SDP1 for some $\alpha \in \overline{\mathrm{A}}$. They also proved that if the feasible region of constraints (2)-(12) is bounded, then the sequence $\left(x^{n}, y^{n}\right)$ is bounded while the sequence $\left\{f^{n}\right\}$ converges to a finite limit. Zymler et al. (2013) indicated that Algorithm 5.1 does not necessarily obtain a global optimal solution to the SDP and SOCP models. However, the methods can perform well in practice. In the next section, we will confirm this point by presenting the numerical results. 


\section{Numerical example}

The purpose of presenting the numerical example in this section is twofold: 1) to demonstrate the effectiveness of the Worst-Case CVaR approximation for distributionally robust joint chance constraints; and 2) to illustrate the advantage of the Worst-Case CVaR approximation approach by comparing with the two other approximation approaches. Under the assumption that the mean and variance of the uncertain demands is known, an example network shown in Figure 1 is selected to test the aforementioned approaches. This cell network is composed of 68 cells and 74 cell connectors. There are three source cells (cells 1, 2, 3) and one sink cell (cell 68). The cells in the center represent the freeway, while the outer and cross cells represent arterial streets. Except the sink cell (cell 68), all cells are considered for capacity expansion. The characteristics of the cells in the test network are shown in Table 2.

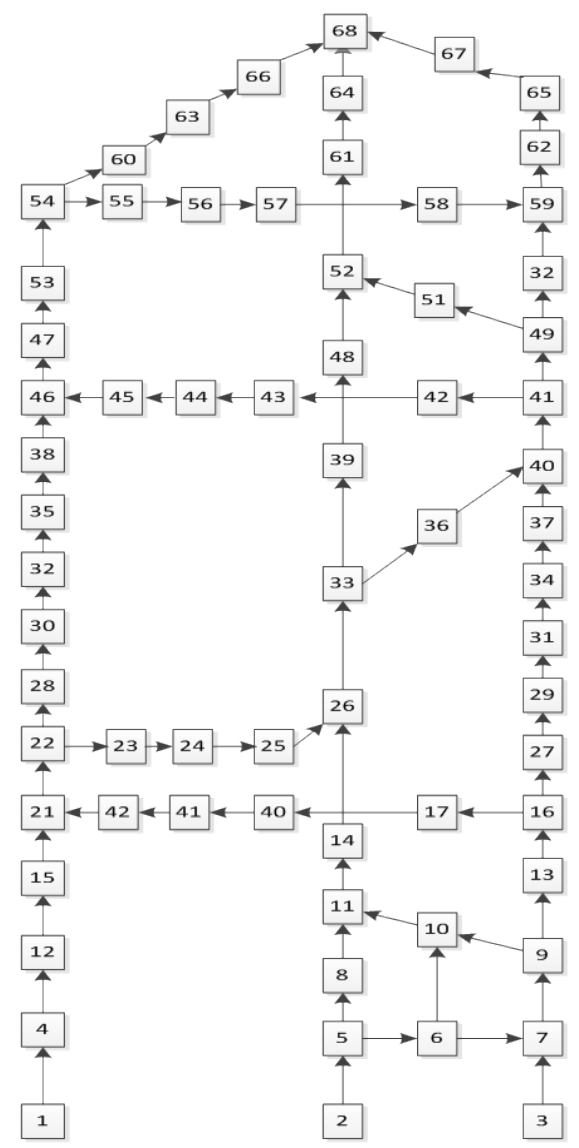

Fig. 1 Test network (Lin et al. 2011)

We assume that the length of a time interval is 1s, the planning horizon is 30s and $M=$ 10. The parameters $\delta$ and $\phi$ are assumed to be unity, i.e., $\delta_{i}^{t}=\phi_{i}=1, \forall i \in C, t \in \mathfrak{I}$.

The mean and variance of the demand are assumed to equal four and one, respectively, i.e., $\mu_{1}^{t}=\mu_{2}^{t}=\mu_{3}^{t}=4, \sigma_{1}^{t}=\sigma_{2}^{t}=\sigma_{3}^{t}=1, \forall t \in \mathfrak{I}$. All second-order cone programs and semidefinite programs arising from the approximation by Chen et al. (2010) and the Worst-Case CVaR approximation were, respectively, solved by using Matlab 7.11 with 
the SeDuMi solver (Sturm 1999) and the YALMIP interface (Löfberg 2004), and the linear programs arising from the Bonferroni approximation was solved by Matlab 7.11 with the GLPK solver and the YALMIP interface (Löfberg 2004).

Table 2 Characteristic of the cells

\begin{tabular}{ccccc}
\hline Cell & $N_{i}^{t}$ & $Q_{i}^{t}$ & $\chi_{i}$ & $\hat{x}_{i}$ \\
\hline 1,3 & $\infty$ & $\infty$ & 1 & 0 \\
2 & $\infty$ & 12 & 1 & 0 \\
68 & $\infty$ & $\infty$ & 1 & 0 \\
$\begin{array}{c}\text { Freeway } \\
\text { Cell }\end{array}$ & 20 & 12 & 1 & 0 \\
Arterial & 10 & 8 & 1 & 0 \\
Cell & & & & 0 \\
\hline
\end{tabular}

Table 3 presents the optimal budget allocations obtained by solving the CTM-based SONDP under the three approximations when $\varepsilon=90 \%$ and $B=50$. This table clearly shows that the total budget is allocated to each cell non-uniformly no matter which approximation method is used. Table 4 describes the optimal objective values and the percentage improvements of the objective values obtained by the SDP approximation relative to the corresponding values obtained by the LP and SOCP approximations under different confidence levels and $B=50$. As expected, the optimal objective value obtained by each of the three approximations increases with $\varepsilon$ because the joint chance constraint becomes more conservative as $\varepsilon$ grows. Moreover, the percentage improvements of the objective values obtained by the SDP approximation relative to those obtained by the LP and SOCP approximations increases with the confidence level $\varepsilon$. When the confidence level $\varepsilon$ approaches 99\%, the SDP approximation outperforms the LP approximation by up to $87 \%$ and the SOCP approximation by up to $0.65 \%$. Moreover, for the same conference level, the SDP approximation yields a smaller optimal objective value than the SOCP approximation, which in turns yields a smaller optimal objective value than the LP approximation.

Table 4 also reports the runtimes required by solving the mathematical programs derived from different approximations. It is obvious to notice that for a fixed conference level, the runtime for solving a linear programs is shorter than that for solving the corresponding second-order cone program, which in turn is shorter than that for solving the corresponding semidefinite program. It is because the problem structure of an LP problem is simpler than that of the corresponding SOCP problem, which is in turn simpler than and that of the corresponding SDP problem. This and the previous observations imply that the improved solution quality offered by the SDP approximation is obtained at the cost of longer computing time.

Table 5 shows the optimal objective values and the percentage improvements of the objective values obtained by the SDP approximation relative to the corresponding values obtained by the LP and SOCP approximations under the different budgets and $\varepsilon=0.9$. It 
can be seen that the optimal objective value obtained by each of the three approximations decreases when the budget increases. It is because the feasible region of the CTM-based SONDP becomes larger as $B$ grows. The two percentage improvements also increase when the budget $B$ increases. When the budget $B$ approaches 70 , the SDP approximation outperforms the LP approximation by up to $85 \%$, and the SOCP approximation by up to $0.27 \%$.

Table 3 Optimal budget allocations to cells obtained by the CTM-based SONDP under the LP, SOCP, and SDP approximations when $\varepsilon=90 \%$ and $B=50$.

\begin{tabular}{cccc}
\hline Cell number & LP & SOCP & SDP \\
\hline 7 & 0 & 1.156 & 1.167 \\
9 & 0 & 1.156 & 1.167 \\
10 & 0 & 1.156 & 1.167 \\
11 & 5.556 & 5.170 & 5.167 \\
14 & 5.556 & 5.170 & 5.167 \\
26 & 5.556 & 5.170 & 5.167 \\
33 & 5.556 & 5.170 & 5.167 \\
39 & 5.556 & 5.170 & 5.167 \\
48 & 5.556 & 5.170 & 5.167 \\
52 & 5.556 & 5.170 & 5.167 \\
61 & 5.556 & 5.170 & 5.167 \\
64 & 5.556 & 5.170 & 5.167 \\
\hline
\end{tabular}

Table 4 The optimal objective values of the CTM-based SONDP under the LP, SOCP, and SDP approximations when $B=50$

\begin{tabular}{|c|c|c|c|c|c|c|c|c|}
\hline \multirow{2}{*}{$\begin{array}{c}\varepsilon \\
(\%)\end{array}$} & \multicolumn{3}{|c|}{ Optimal objective value } & \multirow{2}{*}{$\begin{array}{c}L P-S D P \\
L P \\
(\%)\end{array}$} & \multirow{2}{*}{$\begin{array}{c}\frac{S O C P-S D P}{S O C P} \\
(\%)\end{array}$} & \multicolumn{3}{|c|}{ Runtime } \\
\hline & LP & SOCP & SDP & & & LP & SOCP & SDP \\
\hline 50 & 31521.5361 & 6540.5259 & 6530.0127 & 79.28 & 0.1673 & 352.6791 & 764.3291 & 801.1855 \\
\hline 60 & 34836.3225 & 6877.0969 & 6865.3323 & 80.29 & 0.1710 & 348.9165 & 776.4275 & 827.3901 \\
\hline 70 & 39690.8952 & 7333.1484 & 7319.3851 & 81.56 & 0.1877 & 360.7921 & 791.4784 & 863.2471 \\
\hline 80 & 47829.9268 & 8125.9495 & 8107.3500 & 83.05 & 0.2289 & 367.2473 & 805.2914 & 882.9713 \\
\hline 90 & 66191.1903 & 10071.9018 & 10044.3549 & 84.83 & 0.2737 & 373.9417 & 823.3451 & 905.4637 \\
\hline 95 & 92147.7268 & 12798.5914 & 12759.6528 & 86.15 & 0.3042 & 380.6743 & 834.9186 & 917.7215 \\
\hline 99 & 201660.0676 & 24521.7782 & 24361.4188 & 87.92 & 0.6539 & 392.8234 & 855.1674 & 976.2471 \\
\hline
\end{tabular}


Table 5 The optimal objective values of the CTM-based SONDP under the LP, SOCP, and SDP approximations when $\varepsilon=90 \%$

\begin{tabular}{|c|c|c|c|c|c|c|c|c|}
\hline \multirow[b]{2}{*}{$B$} & \multicolumn{3}{|c|}{ Optimal objective value } & \multirow{2}{*}{$\frac{L P-S D P}{L P}$} & \multirow{2}{*}{$\frac{S O C P-S D P}{S O C P}$} & \multicolumn{3}{|c|}{ Runtime } \\
\hline & LP & SOCP & SDP & & & LP & SOCP & SDP \\
\hline 10 & 66831.1903 & 10631.1680 & 10603.0215 & 84.13 & 0.2648 & 350.8145 & 712.2156 & 767.1963 \\
\hline 20 & 66671.1903 & 10472.8361 & 10444.7993 & 84.33 & 0.2677 & 374.9885 & 724.4762 & 771.8935 \\
\hline 30 & 66511.1903 & 10321.7046 & 10293.6882 & 84.52 & 0.2714 & 383.2667 & 735.6738 & 782.4568 \\
\hline 40 & 66351.1903 & 10185.2395 & 10157.6882 & 84.69 & 0.2705 & 397.2521 & 750.2473 & 789.3479 \\
\hline 50 & 66191.1903 & 10071.9242 & 10044.3549 & 84.83 & 0.2737 & 412.9876 & 776.5429 & 801.1855 \\
\hline 60 & 66031.1903 & 9958.5447 & 9931.0215 & 84.96 & 0.2764 & 434.3687 & 789.4792 & 824.5733 \\
\hline 70 & 65871.1903 & 9845.2362 & 9817.6882 & 85.10 & 0.2798 & 474.2434 & 803.3567 & 851.8935 \\
\hline
\end{tabular}

To compare the operating behaviors of the optimal solutions for the three approximations, we randomly generated 100 travel demand vectors, in which the demand of each O-D pair is uniform distributed between $4-\sqrt{3}$ and $4+\sqrt{3}$. For each random demand vector, the optimal objective values associated with the optimal capacity expansion plans for the three approximations were computed. The mean, standard deviation, and maximum values of the optimal objective values were generated from the simulation experiment. The results are shown and compared in Table 6. It can be seen that the mean, standard deviation, and maximum of the optimal objective values obtained by the Bonferroni (LP) approximation remain unchanged for all confidence levels. This is because that the optimal solutions of Bonferroni approximation are the same under the different confidence levels. Moreover, the mean, standard deviation, and maximum of the optimal objective values obtained by the approximation by Chen et al. (2010) (i.e., the SOCP approximation) and the Worst-Case CVaR approximation (i.e., the SDP approximation) increase with the confidence level $\varepsilon$. This is also because that the joint chance constraints become less restrictive as $\varepsilon$ grows. However, the mean, standard deviation, and maximum of the optimal objective value under the Worst-Case CVaR approximation outperforms the two other approximations. It is continuing to show that the Worst-Case CVaR approximation is less conservative. In addition, when the confidence level $\varepsilon$ increases to a threshold level, the three approximations yield the same mean, standard deviation and maximum of the optimal objective because the optimal solutions for the three approximations are equal.

Table 6 Simulation results of numerical example

\begin{tabular}{ccccccccccc}
\hline & \multicolumn{4}{c}{ Mean } & \multicolumn{4}{c}{ Standard Deviation } & \multicolumn{3}{c}{ Maximum } \\
\cline { 2 - 11 } & LP & SOCP & SDP & LP & SOCP & SDP & LP & SOCP & SDP \\
\hline$B=10$ & & & & & & & & & \\
0.5 & 5229.6119 & 5224.0954 & 5223.8976 & 153.6227 & 153.1981 & 153.1940 & 5606.2974 & 5599.8704 & 5599.6184 \\
\hline
\end{tabular}




\begin{tabular}{|c|c|c|c|c|c|c|c|c|c|}
\hline 0.6 & 5229.6119 & 5225.8138 & 5224.8386 & 153.6227 & 153.2804 & 153.2760 & 5606.2974 & 5602.1704 & 5601.1064 \\
\hline 0.7 & 5229.6119 & 228.7442 & 228.1800 & 153.6227 & 153.3224 & 155.3129 & 5606.2974 & 5604.4764 & 5602.3707 \\
\hline 0.8 & 5229.6119 & 5229.6119 & 5229.6119 & 153.6227 & 153.6227 & 153.6227 & 5606.2974 & 5606.2974 & 5606.2974 \\
\hline 0.9 & 5229.6119 & 5229.6119 & 5229.6119 & 153.6227 & 153.6227 & 53.6227 & 5606.2974 & 5606.2974 & 5606.2974 \\
\hline 0.95 & 229.6119 & 229.6119 & 5229.6119 & 153.6227 & 153.6227 & 153.6227 & 5606.2974 & 5606.2974 & 5606.2974 \\
\hline 0.99 & 5229.6119 & 5229.6119 & 5229.6119 & 153.6227 & 153.6227 & 153.6227 & 5606.2974 & 5606.2974 & 5606.2974 \\
\hline \multicolumn{10}{|l|}{$B=20$} \\
\hline 0.5 & 5210.0316 & 5200.4858 & 5200.3416 & 151.3377 & 149.8323 & 149.8181 & 5582.9654 & 5569.8614 & 5569.6304 \\
\hline 0.6 & 5210.0316 & 5203.2916 & 5202.0731 & 151.3377 & 150.2773 & 150.2498 & 5582.9654 & 5575.1294 & 5574.8434 \\
\hline 0.7 & 5210.0316 & 5206.8949 & 5205.9276 & 151.3377 & 150.7059 & 150.6093 & 5582.9654 & 5580.5474 & 5579.5894 \\
\hline 0.8 & 5210.0316 & 5209.5312 & 5208.5483 & 151.3377 & 151.3260 & 151.3159 & 5582.9654 & 5582.7604 & 5581.7864 \\
\hline 0.9 & 5210.0316 & 5210.0316 & 5210.0316 & 151.3 & 151.3377 & 151.3377 & 5582.9654 & 5582.9654 & 5582.9654 \\
\hline 0.95 & 5210.0316 & 5210.0316 & 5210.0316 & 151.3377 & 151.3377 & 151.3377 & 5582.9654 & 5582.9654 & 5582.9654 \\
\hline 0.99 & & 5210.0 & 5210.0 & 151.3 & 151.3 & 51.3377 & 5582.9654 & 5582.9654 & 5582.9654 \\
\hline \multicolumn{10}{|l|}{$B=30$} \\
\hline 0.5 & 5 & & 5182. & 148 & 546 & & 85 & & 6398 \\
\hline 0.6 & 5193.5155 & 5190.4235 & 5190.2698 & 148.5266 & 147.8264 & 147.7942 & 5560.1585 & 5556.6945 & 5556.4475 \\
\hline 0.7 & 5193.5155 & 5191.2481 & 5190.3737 & 148.5266 & 147.9351 & 147.8379 & 5560.1585 & 5558.0589 & 5557.0989 \\
\hline 0.8 & 5193.5155 & 5192.2688 & 5191.2938 & 148.5266 & 148.1455 & 148.0500 & 5560.1585 & 5559.3389 & 5558.3789 \\
\hline 0.9 & 5193.5155 & 5193.5155 & 5193.5155 & 148.5266 & 148.5266 & 148.5266 & 5560.1585 & 5560.1585 & 5560.1585 \\
\hline 0.95 & 5193.5155 & 5193.5155 & 5193.5155 & 148.5266 & 148.5266 & 148.5266 & 5560.1585 & 5560.1585 & 5560.1585 \\
\hline 0.99 & 5193.5155 & 5193.5155 & 5193.5155 & 148.5266 & 148.5266 & 148.5266 & 5560.1585 & 5560.1585 & 5560.1585 \\
\hline \multicolumn{10}{|l|}{$B=40$} \\
\hline 0.5 & 5180.8740 & 5172.0522 & 5172.0101 & 145.7070 & 142. & 9637 & 958 & 775 & 5528.7295 \\
\hline 0.6 & 5180.8740 & 5176.0302 & 5175.9367 & 145.7070 & 144.3672 & 144.3426 & 5549.7958 & 5532.8504 & 5532.7384 \\
\hline 0.7 & 5180.8740 & 5177.4635 & 5176.2974 & 145.7070 & 144.6887 & 144.6515 & 5549.7958 & 5540.5148 & 5539.2448 \\
\hline 0.8 & 5180.8740 & 5178.5191 & 5177.5539 & 145.7070 & 145.1691 & 145.0771 & 5549.7958 & 5544.4868 & 5543.5448 \\
\hline 0.9 & 5180.8740 & 5180.4647 & 5179.4983 & 145.7070 & 145.6553 & 145.3628 & 5549.7958 & 5549.4688 & 5548.5480 \\
\hline 0.95 & & 5180.8740 & 5180.8740 & 145.7070 & 145.7070 & 145.7070 & 5549.7958 & 49.7958 & 5549.7958 \\
\hline 0.99 & 5180.8740 & 5180.8740 & 5180.8740 & 145.7070 & 145.7070 & 145.7070 & 5549.7958 & 5549.7958 & 5549.7958 \\
\hline \multicolumn{10}{|l|}{$B=50$} \\
\hline 0.5 & 5172.7950 & 5151.2526 & 5150.9999 & 143.2113 & 140.3553 & 140.5882 & 5529.3615 & 5524.4337 & 5523.5655 \\
\hline 0.6 & 5172.7950 & 5168.3943 & 5168.3544 & 143.2113 & 141.5858 & 141.5655 & 5529.3615 & 5526.1495 & 5526.0975 \\
\hline 0.7 & 5172.7950 & 5170.1610 & 5169.8288 & 143.2113 & 142.4167 & 142.0926 & 5529.3615 & 5526.3135 & 5526.2495 \\
\hline 0.8 & 5172.7950 & 5171.1289 & 5170.8262 & 143.2113 & 142.9165 & 142.7945 & 5529.3615 & 5527.4928 & 5526.5288 \\
\hline 0.9 & 5172.7950 & 5172.3447 & 5171.3646 & 143.2113 & 143.0314 & 142.9383 & 5529.3615 & 5528.9916 & 5528.0096 \\
\hline 0.95 & 5172.7950 & 5172.7950 & 5172.7950 & 143.2113 & 143.2113 & 143.2113 & 5529.3615 & 5529.3615 & 5529.3615 \\
\hline 0.99 & 5172.7950 & 5172.7950 & 5172.7950 & 143.2113 & 143.2113 & 143.2113 & 5529.3615 & 5529.3615 & 5529.3615 \\
\hline
\end{tabular}

To illustrate the effect of the means and standard deviations of the uncertain demand on the optimal objective function values under the three approximations, Figure 2 is plotted. The mean and standard deviation of the uncertain demand were set in the range between 4 and 40 and between 1 and 10, respectively and $B=50$ and $\varepsilon=90 \%$. Figure 2 clearly depicts the 
sensitivity of the optimal objective values obtained under the three approximations to the changes in the mean $\mu$ and the standard deviation $\sigma$. In particular, the optimal objective values obtained by the three approximations are all in increasing trend with the mean $\mu$ and the standard deviation $\sigma$. However, the optimal objective values under the Worst-Case CVaR approximation are smaller than the two other approximations under various combinations of the mean $\mu$ and standard deviation $\sigma$.

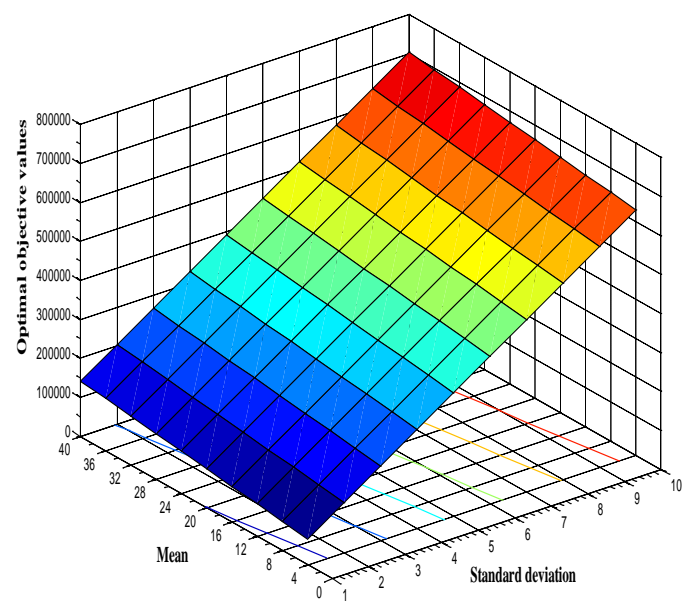

(a) LP approximation

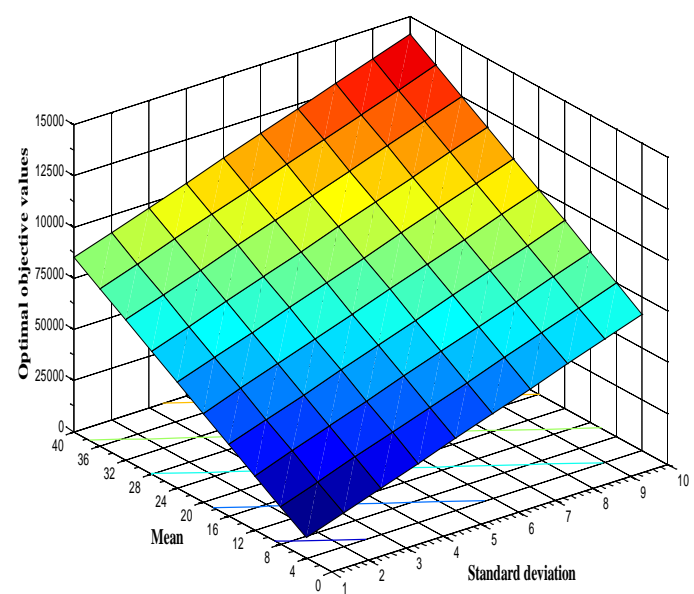

b) SOCP approximation

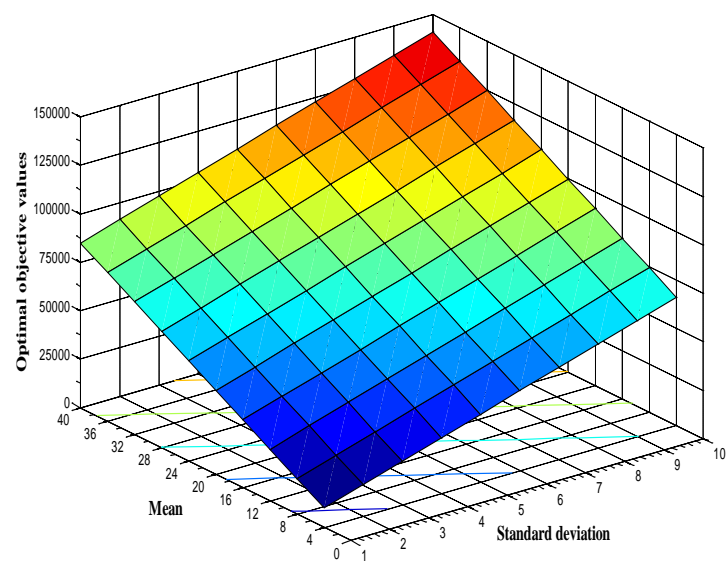

c) SDP approximation

Fig 2 The optimal objective values of the CTM-based SONDP under the LP, SOCP, and SDP approximations under various combinations of the mean and standard deviation of the demand when $B=50$ and $\varepsilon=90 \%$.

Figure 3 further illustrates the percentage improvements of the objective values obtained by the SDP approximation relative to those by the LP and SOCP approximations in the range of the mean and standard deviation used in Figure 2. The percentage improvements increase with an increasing value of the standard deviation $\sigma$, but decrease with an increasing value of the mean $\mu$. This implies that a larger standard deviation yields a larger percentage improvement of the SDP approximation. Thus, the results precisely show that the 
Worst-Case CVaR approximation is more robust and less conservative.

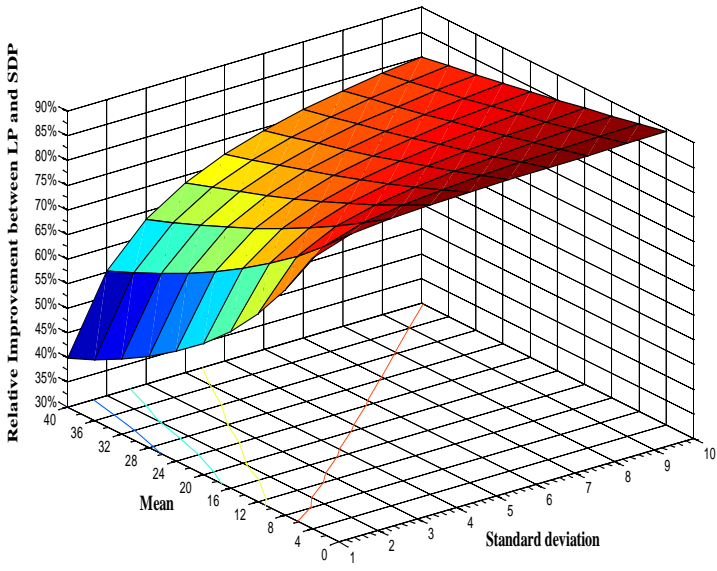

a) SDP vs LP

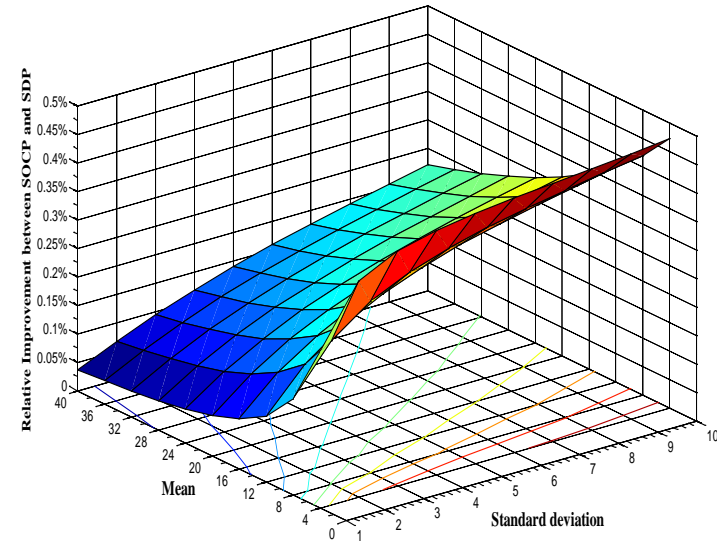

b) SDP vs SOCP

Fig 3 The percentage improvements of the optimal objective values obtained by the SDP approximation relative to those obtained by the LP and SOCP approximations when $B=50$ and $\varepsilon=90 \%$.

\section{Conclusions and future research}

This paper applies the approximation approach for distributionally robust joint chance constraints proposed by Zymler et al. (2013) to the CTM-based SONDP, in which the flow conservation constraint may be violated within a given confidence level due to uncertain demand. Under the assumption that the only partial distribution information, i.e., the mean and variance of the demand, are known, the distributionally robust joint chance constraint of the CTM-based SONDP is approximated by the Worst-Case CVaR constraint, and then reformulated the resultant constraint into the SDP constraint by using the theory of moment problems and conic duality. This paper also presents the two other benchmark approximation approaches and compared the Worst-Case CVaR approximation approach with them. The numerical experiment shows that the Worst-Case CVaR approximation approach outperforms the other two approximation approaches in terms of solution quality.

There are some possible further research directions. Firstly, the classic cell-based SONDP exists holding-back phenomenon (Doan and Ukkusuri, 2012) which may be unrealistic. The Worst-Case CVaR approximation can be applied to the alternative deterministic mathematical formulation (Zhu and Ukkusuri, 2013) to overcome this problem. Secondly, the classic cell-based SONDP only considers demand uncertainty. One possible extension is to replace the CTM by the stochastic CTM (Sumalee et al. 2011) to consider both demand and supply uncertainties and to develop an approximation approach. Thirdly, the Worst-Case CVaR approximation has only been applied to the studied SONDP, which is a single-level optimization problem. How to apply Worst-Case CVaR approximation to the dynamic user equilibrium NDP is another challenging research direction because the problem is bi-level by nature. Lastly, the assumption that the mean and variance of the demand are known can be relaxed. The approach proposed by Delage and Ye (2010) may be used to solve the CTM-based NDP when the mean and variance of the demand are unknown but bounded. 


\section{Acknowledgments}

The work described in this paper was jointly supported by grants from the National Natural Science Foundation of China (71131001, 71271075, 71271183), the National Basic Research Program of China (2012CB725400), and the Program for New Century Excellent Talents in University (NCET-13-0766). The authors are grateful to the referees for their constructive comments.

\section{References}

Ben-Tal A, Nemirovski A (1998) Robust convex optimization. Math Oper Res 23(4):769-805.

Ben-Tal A, Nemirovski A (1999) Robust solutions of uncertain linear programs. Oper Res Lett 25(1):1-13.

Ben-Tal A, Nemirovski A (2000) Robust solutions of linear programming problems contaminated with uncertain data. Math Program 88(3):411-424.

Ben-Tal A, Nemirovski A (2002) Robust optimization-methodology and applications. Math Program 92(3): 453-480.

Ben-Tal A, Goryashko A, Guslitzer E, Nemirovski A (2004) Adjustable robust solutions of uncertain linear programs. Math Program 99 (2):351-376.

Ben-Tal A, El Ghaoui L, Nemirovski A (2009) Robust Optimization. Princeton Series in Applied Mathematics, Princeton University Press.

Ben-Tal A, Chung BD, Mandala SR, Yao T (2011) Robust optimization for emergency logistics planning: Risk mitigation in humanitarian relief supply chains. Transp Res B 45(8):1177-1189.

Bertsimas D, Sim M (2004) The price of robustness. Oper Res 52(1): 35-53.

Bertsimas D, Brown DB, Caramanis C (2011) Theory and applications of robust optimization. SIAM Rev 53(3):464-501.

Boyce DE (1984) Urban transportation network-equilibrium and design models: Recent achievements and future prospects. Environ Plann A 16(11):1445-1474.

Calafiore GC, El Ghaoui L. (2006) On distributionally robust chance-constrained linear programs with applications. J Optim Theory Appl 130(1):1-22.

Charnes A, Cooper WW, Symonds GH (1958) Cost horizons and certainty equivalents: an approach to stochastic programming of heating oil. Manag Sci 4(3):235-263.

Chen A, Zhou Z, Chootinan P, Ryu S, Yang C, Wong SC (2011) Transportation network design problem under uncertainty: a review and new developments. Transp Rev 31(6):743-768.

Chen W, Sim M (2009) Goal-driven optimization. Oper Res 57(2):342-357.

Chen W, Sim M, Sun J, Teo CP (2010) From CVaR to uncertainty set: implications in joint chance-constrained optimization. Oper Res 58(2):470-485.

Chung BD, Yao T, Xie C, Thorsen A (2011) Robust optimization model for a dynamic design problem under demand uncertainty. Netw Spat Econ 11(2):371-389.

Chung BD, Yao T, Zhang B (2012) Dynamic traffic assignment under uncertainty: A distributional robust chance-constrained approach. Netw Spat Econ 12(1):167-181. 
Daganzo CF (1994) The cell transmission model: a dynamic representation of highway traffic consistent with the hydrodynamic theory. Transp Res B 28(4):269-287.

Daganzo CF (1995) The cell transmission model, part II: network traffic. Transp Res B 29(2):79-93. Janson BN (1995) Network design effects of dynamic traffic assignment. J Transp Eng 121(1):1-13. Delage E, Ye Y (2010) Distributionally robust optimization under moment uncertainty with application to data-driven problems. Oper Res 58(3):595-612.

Doan K, Ukkusuri SV (2012) On the holding-back problem in the cell transmission based dynamic traffic assignment models. Trans Res B 46(9):1218-1238.

Karoonsoontawong A, Waller ST (2006) Dynamic continuous network design problem: linear bilevel programming and metaheuristic approaches. Transp Res Rec: J Transp Res Board 1964:104-117.

Karoonsoontawong A, Waller ST (2007) Robust dynamic continuous network design problem. Transp Res Rec: J Transp Res Board 2029:58-71.

Karoonsoontawong A, Waller ST (2010) Integrated network capacity expansion and traffic signal optimization problem: robust bi-level dynamic formulation. Netw Spat Econ 10(4):525-550.

Kouvelis P, Yu G (1997) Robust Discrete Optimization and Its Applications. Kluwer Academic Publishers, Boston, USA.

Li Y, Waller ST, Ziliaskopoulos T (2003) A decomposition scheme for system optimal dynamic traffic assignment models. Netw Spat Econ 3(4):441-455.

Lin DY, Karoonsoontawong A, Waller ST (2011) A Dantzig-Wolfe decomposition based heuristic scheme for bi-level dynamic network design problem. Netw Spat Econ 11(1):101-126.

Lin DY (2011) A dual variable approximation-based descent method for a bi-level continuous dynamic network design problem. Comput Aided Civil Infrastruct Eng 26(8):581-594.

Löfberg J (2004) YALMIP: a toolbox for modeling and optimization in MATLAB. In Proceedings of the CACSD conference, Taipei.

Lou Y, Yin Y, Lawpongpanich S (2009) Robust approach to discrete network designs with demand uncertainty. Transp Res Rec: J Transp Res Board 2090:86-94.

Lou Y, Yin Y, Lawpongpanich S (2010) Robust congestion price under rational user equilibrium. Transp Res B 44(1): 15-28.

Magnanti TL, Wong RT (1984) Network design and transportation planning: models and algorithms. Transp Sci 18(1):1-15.

Minoux M (1989) Network synthesis and optimum network design problems: models, solution methods and applications. Netw 19(3):313-360.

Mudchanatongsuk S, Ordóñez F, Liu J (2008) Robust solutions for network design under transportation cost and demand uncertainty. J Oper Res Soc 59(5):652-662

Mulevy JM, Vanderbei RJ, Zenios SA (1995) Robust optimization of large-scale systems. Oper Res 43(2):264-281.

Nemirovski A, Shapiro A (2006) Convex approximations of chance constrained programs. SIAM J Optim 17(4):969-996. 
Patil GR, Ukkusuri SV (2007) System-optimal stochastic transportation network design. Transp Res Rec: J Transp Res Board 2029:80-86.

Rockafellar RT, Uryasev S (2000) Optimization of conditional value-at-risk. J Risk 2(3): 21-41.

Rockafellar RT, Uryasev S (2002) Conditional Value-at-Risk for general loss distributions. J Bank Finance 26(7): 1443-1471.

Sharipo A, Kleywegt AJ (2002) Minimax analysis of stochastic problems. Optim Methods Softw 17(3):523-542.

Sturn JF (1999) Using SeDuMi 1.02, a Matlab toolbox for optimization over symmetric cones. Optim Methods Softw 11(1-4): 625-653.

Sumalee A, Zhong RX, Pan TL, Szeto WY (2011) Stochastic cell transmission model (SCTM): a stochastic dynamic traffic model for traffic state surveillance and assignment. Transp Res B 45(3):507-533.

Ukkusuri SV, Mathew TV, Waller ST (2007) Robust Networks Design model using multi-objective evolutionary algorithms. Comput Aided Civil Infrastruct Eng 22(1):9-21.

Ukkusuri SV, Waller ST (2008) Linear programming models for the user and system optimal dynamic network design problem: formulations, comparisons and extensions. Netw Spat Econ 8(4):383-406.

Waller ST (2000) Optimization and control of stochastic dynamic transportation systems: formulations, solution methodologies, and computational experience. Ph.D. Dissertation, Northwestern University.

Waller ST, Ziliaskopoulos AK (2001) Stochastic dynamic network design problem. Transp Res Rec: J Transp Res Board 1771:106-113.

Waller ST, Schofer JL, Ziliaskopoulos AK (2001). Evaluation with traffic assignment under demand uncertainty. Transp Res Rec: J Transp Res Board 1771: 69-74.

Waller ST, Ziliaskopoulos AK (2006) A chance-constrained based stochastic dynamic traffic assignment model: analysis, formulation and solution algorithms. Transp Res C 14(6):418-427.

Waller ST, Mousokos KC, Kamaryiannis D, Ziliaskopoloulos AK (2006) A linear model for the continuous network design problem. Comput Aided Civil Infrastruct Eng 21(5):334-345.

Yang H, Bell MGH (1998) Models and algorithms for road network design: a review and some new developments. Transp Rev 18(3):257-278.

Yao T, Mandala SR, Chung BD (2009) Evacuation transportation planning under uncertainty: a robust optimization approach. Netw Spat Econ 9(2):171-189.

Yazici A, Ozbay K (2010) Evacuation network modeling via dynamic traffic assignment with probability demand and capacity constraints. Transp Res Rec: J Transp Res Board 2196:11-20.

Yin Y, Lawpongpanich S (2007) A robust approach to continuous network designs with demand uncertainty. Proc $17^{\text {th }}$ Int Sympo Transp Traffic Theory 111-126.

Yin Y, Lawpongpanich S (2008) Estimating investment requirement for maintaining and improving highway systems. Transp Res C 16(2): 199-211.

Yin Y, Madanat SM, Lu XY (2009) Robust improvement schemes for road networks under demand uncertainty. Euro J Oper Res 198(2):470-479.

Zhao Y, Kockelman KM (2002) The propagation of uncertainty through travel demand models: an 
exploratory analysis. Ann Reg Sci 36(1):145-163.

Zhu F, Ukkusuri SV (2013) A cell based dynamic system optimum model with non-holding back flows. Transp Res C 36:367-380.

Ziliaskopoulos AK (2000) A linear programming model for the single destination system optimum dynamic traffic assignment problem. Transp Sci 34(1):37-49.

Zymler S, Kuhn D, Rustem B (2013) Distributionally robust joint chance constraints with second-order moment information. Math Program 137(1-2):167-198. 